\title{
Left clavicular fracture
}

\author{
Lulu He $^{1}$ (D) Carl S. Winalski ${ }^{2} \cdot$ Chad Deal $^{3} \cdot$ Darlene Holden $^{4}$
}

Published online: 5 January 2016

(C) ISS 2016

\section{ANSWER: Pyknodysostosis}

Pyknodysostosis, also known as Maroteaux-Lamy disease and Toulouse-Lautrec syndrome, is a rare autosomal recessive genetic sclerosing bone dysplasia with an estimated incidence of 1.7 per million births with no gender predilection [1]. The disorder is caused by a mutation of a lysosomal cysteine proteinase, cathepsin $\mathrm{K}$, that is required for normal degradation of type I collagen, the major constituent ( $95 \%$ ) of organic bone matrix $[1,2]$. The resultant osteoclast dysfunction impairs bone remodeling with resultant dense but fragile bones. Although a cathepsin $\mathrm{K}$ gene mutation analysis can provide confirmation, the diagnosis is usually based on the characteristic clinical and radiographic findings [3].

Clinically, patients demonstrate short-stature measuring $<150 \mathrm{~cm}$ (4 feet 11 inches) and dysmorphic facial features including a beaked nose and micrognathia. Underlying bone

The case presentation can be found at doi: 10.1007/s00256-015-2318-y

Lulu $\mathrm{He}$

HEL@ccf.org

1 Department of Diagnostic Radiology, Imaging Institute, Cleveland Clinic, Mail Code L10, 9500 Euclid Ave, Cleveland, OH 44195, USA

2 Department of Diagnostic Radiology, Imaging Institute, Cleveland Clinic, Mail Code A21, 9500 Euclid Avenue, Cleveland, OH 44195, USA

3 Department of Rheumatologic and Immunologic Disease, Orthopaedic and Rheumatologic Institute, Cleveland Clinic, Mail Code A50, 9500 Euclid Avenue, Cleveland, OH 44195, USA

4 Department of Diagnostic Radiology, Imaging Institute, Cleveland Clinic, Mail Code AC116, 9500 Euclid Avenue, Cleveland, OH 44195, USA fragility is clinically manifest as multiple fractures after only minor trauma [4]. It is believed that the famous French postimpressionist painter, Henri de Toulouse-Lautrec had this disorder and suffered from bilateral femoral fractures at a young age from falls [5]. Typical locations for pathologic fracture include the jaw, clavicles, and lower extremities. Because of impaired vascularity in the dense bones, there is a propensity to develop jaw osteomyelitis after dental extraction or injury that can be refractory to treatment [6]. Affected individuals are usually diagnosed at a young age because of the characteristic appearance and bone fragility, but in some instances, as in this case, the diagnosis is made later in life. Cognitive function and life expectancy are normal [1].

Radiographic findings include generalized osteosclerosis with preservation of the medullary canal of long bones. For our patient, the osteosclerosis was not radiographically obvious but the DEXA scan detected increased bone density. There is foreshortening of the distal phalanges with irregular terminal tufts simulating acro-osteolysis, a finding considered a pathognomonic feature [4]. The calvarium demonstrates open fontanelles, widened sutures and, often times, wormian bones. There is also hypoplasia of the paranasal sinuses and an obtuse mandibular angle. Less commonly there is a thoracic deformity including kyphosis and pectus excavatum and distal clavicular hypoplasia. Segmentation abnormalities and spondylolisthesis in the cervical or lumbar spine can also be seen [7]. Long bone fractures are in the midshaft and are typically transverse rather than oblique, as with other pathological bone fractures.

The radiological differential diagnosis includes osteopetrosis, which also presents with brittle sclerotic bones; however, with osteopetrosis, sutures of the skull are closed and there is typically obliteration of the medullary canal of the long bones with resultant anemia. Cleidocranial dysplasia may have some similar imaging features including open 
fontanelles and wormian bones; nevertheless, there should not be increased bone density and the clavicles are typically hypoplastic. The appearance of the hands of our patient could raise consideration of other causes of acro-osteolysis; however, the other imaging features are characteristic of pyknodysostosis.

Management of patients with pyknodysostosis is primarily supportive. Factures are treated conservatively or with internal fixation if necessary. Special consideration should be given to patients undergoing general anesthesia due to anticipated difficult intubation and cervical spine anomalies [8]. In conclusion, pyknodysostosis should be considered in the differential diagnosis of patients with elevated bone mineral density and fragility fractures. While unusual, the disorder may go unrecognized until adulthood and be first diagnosed by radiological findings.

\section{Compliance with ethical standards}

Conflict of interest The authors declare that they have no conflict of interest.

\section{References}

1. Bernard B, Hiddema W. Pycnodysostosis with the focus on clinical and radiographic findings. S Afr J Rad. 2012;16:74-6.

2. Gelb BD, Shi GP, Chapman HA, Desnick RJ. Pycnodysostosis, a lysosomal disease caused by cathepsin K deficiency. Science. 1996;273(5279):1236-8.

3. Ihde LL, Forrester DM, Gottsegen CJ, et al. Sclerosing bone dysplasia: review and differentiation from other cause of osteosclerosis. Radiographics. 2011;31:1865-82.

4. Beighton P, Horan F, Hamersma H. A review of the osteopetroses. Postgrad Med J. 1977;53:507-16.

5. Hodder A, Huntley C, Aronson JK, Ramachandran M. Pycnodysostosis and the making of an artist. Gene. 2015;555:59-62.

6. Bathi RJ, Masur VN. Pyknodysostosis: a report of two cases with a brief review of the literature. Int J Oral Maxillofac Surg. 2000;29: 439-42.

7. Herring JA. Tachdjian's pediatric orthopaedics: from the Texas Scottish Rite Hospital for Children. Philadelphia: Elsevier; 2014.

8. Puri R, Saxena A, Mittal A, et al. Pycnodysostosis: an anaesthetic approach to this rare genetic disorder. Case Rep Anesthesiol. 2013;2013:716756. 